\title{
THE CAUCHY PROBLEM FOR SYMMETRIC HYPERBOLIC SYSTEMS IN $L_{p}$
}

\section{PHILIP BRENNER}

\section{Introduction.}

It is well known that the initial-value problem for a symmetric hyperbolic system,

$$
\begin{cases}\frac{\partial u}{\partial t}=\sum_{j=1}^{n} A_{j} \frac{\partial u}{\partial x_{j}}+B u, & x \in R^{n}, \\ u(0, x)=u_{0}(x), & 0 \leqq t \leqq T,\end{cases}
$$

is well posed in $L_{2}$. The purpose of this note is to prove that the problem (1) is well posed in $L_{p}, p \neq 2,1 \leqq p \leqq \infty$, if and only if the matrices $A_{j}$ commute (Theorem 2). This will be proved by noticing that, a necessary and sufficient condition for (1) to be well posed in $L_{p}$ is that $\exp \left(i \sum_{j=1}^{n} A_{j} y_{j}\right)$ is a multiplier on $L_{p}$ which in turn will be proved to be the case if and only if the $A_{j}$ commute (Theorem 1). This last statement follows by application of the technique developed by Hörmander in [1] (frequent references will be made to that paper) and a matrix theorem by Motzkin and Taussky [3].

The corresponding problem for the wave operator has been treated by Littman [2]; his result is included in ours.

I wish to thank Professor Vidar Thomée for suggesting the problem and for several helpful discussions.

\section{Multipliers on $\boldsymbol{L}_{p}$.}

First some notation. If $v=\left(v_{1}, \ldots, v_{N}\right)$ and $u=\left(u_{1}, \ldots, u_{N}\right)$ are complex vectors, $\langle u, v\rangle$ will denote their scalar product and $|v|$ the Euclidean norm,

$$
\langle u, v\rangle=\sum_{j=1}^{N} u_{j} \bar{v}_{j}, \quad|v|=\left(\sum_{j=1}^{N}\left|v_{j}\right|^{2}\right)^{\frac{1}{2}} .
$$

The norm $|A|$ of an $N \times N$-matrix $A$ is defined as

$$
|A|=\sup \left\{|A v| ; v=\left(v_{1}, \ldots, v_{N}\right),|v| \leqq 1\right\} .
$$

Received March 9, 1966. 
If $\Omega \subset R^{n}$ is open and $v_{j} \in C^{\infty}(\Omega)$ for $j=1, \ldots, N$, then we say that $v=\left(v_{1}, \ldots, v_{N}\right)$ belongs to $\mathscr{C}^{\infty}(\Omega)$. If $g \in C^{\infty}\left(R^{n}\right)$, and if

$$
\sup \left\{|x|^{m}\left|D^{k} g(x)\right| ; x \in R^{n}\right\}<\infty
$$

for $m=0,1, \ldots$ and for any multi-index $k=\left(k_{1}, \ldots, k_{n}\right)$,

$$
D^{k}=\left(\partial^{k_{1}} / \partial x_{1}^{k_{1}}\right) \ldots\left(\partial^{k_{n}} / \partial x_{n}{ }^{k_{n}}\right),
$$

then we say that $g$ belongs to $S$. We give the linear space $S$ the topology defined by the family (2) of semi-norms. We denote by $\mathscr{S}$ the set of functions $v=\left(v_{1}, \ldots, v_{N}\right)$ with $v_{j} \in S, j=1, \ldots, N$. The dual space $S^{\prime}$ of $S$ is the set of tempered distributions (in the sense of Schwartz).

The convolution between a tempered distribution $\mu$ and a function $g \in S$ is denoted by $\mu * g$, and defined by $\mu(g(x-\cdot))=\mu * g(x)$. This notion also has an obvious meaning if $g$, say, is replaced by a vector in $\mathscr{S}$. We can then also replace $\mu$ by an $N \times N$-matrix, the elements of which are tempered distributions. The Fourier transform of a tempered distribution $\mu$ is denoted by $\hat{\mu}$, and defined by $\hat{\mu}(f)=\mu(\hat{f}), f \in S$, where $\hat{f}$ is the function

$$
\hat{f}(y)=\int_{R^{n}} \exp (2 \pi i\langle x, y\rangle) f(x) d x .
$$

The Fourier transform is also defined for matrices and vectors of tempered distributions by applying the transform elementwise.

By $\mathscr{L}_{p}$ we means the set of functions $v=\left(v_{1}, \ldots, v_{N}\right)$ with $v_{j} \in L_{p}$, $j=1, \ldots, N$, and for $p<\infty$ we set

and for $p=\infty$

$$
\|v\|_{p}=\left(\int_{R^{n}}|v(x)|^{p} d x\right)^{1 / p}
$$

$$
\|v\|_{\infty}=\operatorname{ess} \sup \left\{|v(x)| ; x \in R^{n}\right\} .
$$

Classically a multiplier on $L_{p}$ is a function $\lambda$ such that for each $f \in L_{p}$, $\lambda \hat{f}$ is the Fourier transform of a function in $L_{p}$. Following Hörmander [1] we formalize this as follows: We say that $\lambda$ is a multiplier on $L_{p}, \lambda \in M_{p}$, if $\lambda \in S^{\prime}$ and if

$$
M_{p}(\lambda)=\sup \left\{\|\hat{\lambda} * f\|_{p} ; f \in S,\|f\|_{p} \leqq 1\right\}<\infty .
$$

We will need the following natural generalization to matrices: We define $\mathscr{M}_{p}$, the multipliers on $\mathscr{L}_{p}$, as the set of $N \times N$-matrices $\mu$ with elements in $S^{\prime}$ satisfying

$$
\mathscr{H}_{p}(\mu)=\sup \left\{\|\hat{\mu} * v\|_{p} ; v \in \mathscr{S},\|v\|_{p} \leqq 1\right\}<\infty .
$$


Since the norms $\|v\|_{p}$ and $\sup _{j}\left\|v_{j}\right\|_{p}$ are equivalent, this definition can also be expressed by saying that $\mu=\left(\mu_{j k}\right) \in \mathscr{M}_{p}$ if $\mu_{j k} \in M_{p}, j, k=1, \ldots, N$.

In order to get shorter statements it will be convenient to define $M_{p}(\lambda)=\infty$ if $\lambda \notin M_{p}$, and correspondingly for $\mathscr{M}_{p}$. Thus $\mu \in \mathscr{M}_{p}$ if and only if $\mathscr{M}_{p}(\mu)<\infty$.

We collect some facts about $\mathscr{M}_{p}$ in the following lemma.

Lemma 1. Suppose $1 \leqq p \leqq \infty$. Then

(i) $\mathscr{M}_{p}=\mathscr{M}_{q}, 1 / p+1 / q=1$, and $\mathscr{M}_{1} \subset \mathscr{M}_{p} \subset \mathscr{M}_{2}$.

(ii) $\mathscr{M}_{p}$ is a Banach algebra under pointwise (matrix-) multiplication and addition, with the norm $\mathscr{M}_{p}(\cdot)$. It is non-commutative for $N>1$.

(iii) $\mathscr{M}_{2}$ is the set of essentially bounded $N \times N$-matrices, and $\mathscr{M}_{2}(\cdot)=$ ess $\sup |\cdot| . \quad \mathscr{M}_{1}$ is the set of $N \times N$-matrices, the elements of which are Fourier-Stieltjes transforms of bounded measures.

(iv) Suppose $y_{0} \in R^{n}$ and $a \in R-\{0\}$ and let $a^{*} f(y)=f(a y)$ and $f_{y_{0}}(y)=$ $f\left(y+y_{0}\right)$. Then $\mathscr{M}_{p}(f)=\mathscr{M}_{p}\left(a^{*} f\right)=\mathscr{M}_{p}\left(f_{y_{0}}\right)$.

(v) Let $\mathscr{M}_{p}\left(f_{i}\right) \leqq C$, all $i \in I$, and suppose $f_{i} \rightarrow f$ in $S^{\prime}$ (e.g. uniformly on compact subsets of $\left.R^{n}\right)$. Then $\mathscr{M}_{p}(f) \leqq C$.

(vi) Let $\alpha_{j} \in R, j=0,1, \ldots, n$, and $\alpha(y)=\alpha_{0}+\sum_{j=1}^{n} \alpha_{j} y_{j}$. Then $M_{p}(\exp (i \alpha))=1$.

(vii) If $k \in S$, then $\mathscr{M}_{p}(k E) \leqq\|\hat{k}\|_{1}$, where $E$ is the unite matrix.

Proof. For the case $N=1$ these statements are all contained in Chapter I of [1]. Most of the generalisations to $N>1$ are obvious. Below we will just give references to the corresponding statements in [1] for those cases.

(i) For $N=1$ this is Theorem 1.3 in [1].

(ii) Corollary 1.4 in [1].

(iii) Theorem 1.4 and 1.5 in [1].

(iv) Theorem 1.13 in [1]. See also Lemma 3(iii) below.

(v) By Hölders inequality we have $(1 / p+1 / q=1)$

$$
\left|\int\left\langle\hat{f}_{i} * u(x), v(x)\right\rangle d x\right| \leqq C\|u\|_{p}\|v\|_{q}, \quad u, v \in \mathscr{S} .
$$

Since $f_{i} \rightarrow f$ in $S^{\prime}$ implies that $\hat{f}_{i} \rightarrow \hat{f}$ in $S^{\prime}$, we see that also $\hat{f}$ satisfies this inequality. The converse of Hölders inequality then gives

that is, $\mathscr{M}_{p}(f) \leqq C$.

$$
\|\hat{f} * u\|_{p} \leqq C\|u\|_{p}, \quad u \in \mathscr{S},
$$

(vi) Multiplying $\hat{a} \in S$ by $\exp (i \alpha)$ corresponds to a translation of $u$ with $\left(\frac{1}{2} \pi\right)\left(\alpha_{1}, \ldots, \alpha_{n}\right)$ followed by multiplication with $\exp \left(i \alpha_{0}\right)$, and hence $M_{p}(\exp (i \alpha))=1$. 
(vii) This follows from the inequality

$$
\|\hat{k} * u\|_{p} \leqq\|\hat{k}\|_{1}\|u\|_{p}, \quad k \in S, u \in \mathscr{S} \text { (or } k \in \mathscr{S}, u \in S \text { ), }
$$

which is proved just as in the scalar case.

We want to study functions which are locally multipliers on $\mathscr{L}_{p}$ and so make the following definition: Let $B$ be an open ball in $R^{n}$ (the open ball with center $x$ and radius $r$ will be denoted $B(x, r))$. We say that an $N \times N$-matrixfunction $\varphi$ is an $\mathscr{L}_{p}$-multiplier on $B, \varphi \in \mathscr{M}_{p, B}$, if there is a $\mu \in \mathscr{M}_{p}$ such that $\varphi=\mu$ on $B$. If $\varphi \in \mathscr{M}_{p, B}$ and $\varphi=\mu$ on $B, \mu \in \mathscr{M}_{p}$, we can define

$$
\mathscr{M}_{p, B}(\varphi)=\sup \left\{\|\hat{\mu} * \hat{\imath}\|_{p} ; u \in \mathscr{S}, u=0 \text { outside } B,\|\hat{u}\|_{p} \leqq 1\right\}
$$

since $\mu u$ does not depend on the behavior of $\mu$ outside $B$. We note that $\mathscr{M}_{p, B}(\cdot)$ is an semi-norm, and that $\mathscr{M}_{p, R^{n}}(\cdot)=\mathscr{M}_{p}(\cdot)$. For $N=1$ we write $M_{p, B}$ and $M_{p, B}(\cdot)$.

The following well known lemma will be useful in this context.

Lemma 2. Suppose that $B$ is a bounded open ball in $R^{n}$ and $\varepsilon$ a positive number. Then there is a function $k \in S$ such that $k=1$ on $B, k$ has compact support and $\|\hat{k}\|_{1} \leqq 1+\varepsilon$.

Proof. We can suppose that $B=B(0, r)$. Let $m(r)$ be the volume of $B(0, r)$. Choose $\varrho$ so that $m(r+\varrho)+1 \leqq(1+\varepsilon)^{2} m(\varrho)$, and let $g$ be the characteristic function of $B(0, \varrho)$. Further choose $h \in S$, such that $h=1$ on $B(0, r+\varrho)$ and $h=0$ outside $B(0, r+2 \varrho)$ and

Set

$$
\int|h(y)|^{2} d y \leqq m(r+\varrho)+1 .
$$

$$
k(y)=(m(\varrho))^{-1} h * g(y) \text {. }
$$

Then $k \in C^{\infty}\left(R^{n}\right), k=1$ on $B(0, r)$ and has compact support. By Schwartz' inequality and Parsevals formula

$$
\|\hat{\mathcal{k}}\|_{1} \leqq(m(\varrho))^{-1}\|h\|_{2}\|g\|_{2} \leqq\left(\frac{m(r+\varrho)+1}{m(\varrho)}\right)^{1} \leqq 1+\varepsilon
$$

and so $k$ is the desired function.

We can now give some facts about $\mathscr{M}_{p, B}$.

Lemma 3. Suppose $1 \leqq p \leqq \infty$. Then

(i) if $B \subset B^{\prime}$, then $\mathscr{M}_{p, B}(\varphi) \leqq \mathscr{M}_{p, B^{\prime}}(\varphi)$.

(ii) if $a \in R-\{0\}$ and $y_{0} \in R^{n}$, then $\mathscr{M}_{p, B}(\varphi)=\mathscr{M}_{p, a^{-1 B}}\left(a^{*} \varphi\right)=$ $\mathscr{M}_{p, B-\nu_{0}}\left(\varphi_{\nu_{0}}\right)$. 
(iii) $\mathscr{M}_{p, B}(\nu \varphi) \leqq \mathscr{M}_{p}(v) \mathscr{M}_{p, B}(\varphi)$ and if $k \in S$, then $\mathscr{M}_{p, B}(k \varphi) \leqq$ $\mathscr{M}_{p, B}(\varphi)\|\hat{k}\|_{1}$.

(iv) if $\mathscr{M}_{p, B}(\varphi) \leqq C$ for all bounded open balls in $R^{n}$, then $\mathscr{M}_{p}(\varphi) \leqq C$.

Proof. (i) Obvious.

(ii) We note that a change of coordinates in $R^{n}$ only changes the

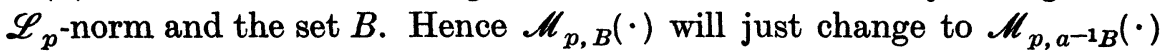
under the transformation $y \rightarrow a y$. If $\varphi=\mu$, on $B, \mu \in \mathscr{M}_{p}$, then let $\mu_{1}=$ $\mu_{y_{0}}$, and so $\mu_{1}=\varphi_{y_{0}}$ on $B-y_{0}$. It follows that $\hat{\mu}_{1}=\exp \left(2 \pi i\left\langle\cdot, y_{0}\right\rangle\right) \hat{\mu}$. Since multiplication with a scalar function of absolute value 1 is an isometry on $\mathscr{L}_{p}$, we see that $\mathscr{M}_{p, B}(\varphi)=\mathscr{M}_{p, B-y_{0}}\left(\varphi_{y_{0}}\right)$.

(iii) The first assertion follows from Lemma l(ii) and the definitions. The second is an application of this, using Lemma 1 (vii).

(iv) Let $\varepsilon>0$ be arbitrary. Choose a sequence $\left\{B_{j}\right\}_{1}^{\infty}$ of bounded open balls and functions $k_{j}$ such that (a) $\bar{B}_{j} \subset B_{j+1}$ and $\bigcup_{j=1}^{\infty} B_{j}=R^{n}$, (b) $k_{j}=1$ on $B_{j}$ and $k_{j}=0$ outside $B_{j+1}$, (c) $\left\|\hat{k}_{j}\right\|_{1} \leqq 1+\varepsilon$. This is possible by Lemma 2 . Let $\varphi=\mu_{j}$ on $B_{j+1}, \mu_{j} \in \mathscr{M}_{p}$. Let $v_{j}=\mu_{j} k_{j}$. Then by (3)

$\mathscr{M}_{p}\left(v_{j}\right)=\sup \left\{\left\|\hat{\mu}_{j} * \hat{k}_{j} * \hat{f}\right\|_{p} ; f \in \mathscr{S},\|f\|_{p} \leqq 1\right\} \leqq \mathscr{M}_{p, B_{j+1}}(\varphi)\left\|\hat{k}_{j}\right\|_{1} \leqq(1+\varepsilon) C$.

Since $v_{j} \rightarrow \varphi$ uniformly on compact subsets of $R^{n}$, Lemma 1 (iv) gives that $\mathscr{M}_{p}(\varphi) \leqq(1+\varepsilon) C$. As $\varepsilon>0$ was arbitrary (iv) is proved.

We will now state the main theorem of this section.

Theorem 1. Suppose $1 \leqq p \leqq \infty$ and $p \neq 2$. Let $A_{j}$ be Hermitian $N \times N$ matrices $(j=1, \ldots, n)$. Then $\exp \left(i \sum_{j=1}^{n} A_{j} y_{j}\right)$ belongs to $\mathscr{M}_{p}$ if and only if the matrices $A_{1}, \ldots, A_{n}$ commute.

We need some lemmas for the necessity part of the proof.

Lemma 4. Let $B=B\left(x_{0}, r\right), r>0$. If $v \in \mathscr{S}$ and $v \neq 0$ on $B$, then there is a constant $C$ and $a$ ball $B^{\prime}=B\left(x_{0}, r^{\prime}\right), 0<r^{\prime} \leqq r$, such that for each $g \in S$ with $g=0$ outside $B^{\prime}$, we have

$$
\|\hat{g}\|_{p} \leqq C\|\hat{v} * \hat{g}\|_{p} .
$$

Proof. Since $v$ is continuous, there is a $k, 1 \leqq k \leqq N$, and a ball $B^{\prime}=$ $B\left(x_{0}, r^{\prime}\right), 0<r^{\prime} \leqq r$, such that $v_{k} \neq 0$ on $\bar{B}^{\prime}$. Hence there is a $w_{k} \in S$ such that $w_{k} v_{k}=1$ on $B^{\prime}$. We get for any $g \in S$ with $g=0$ outside $B^{\prime}$

and so the inequality (3) gives

$$
g=w_{k} v_{k} g
$$

$$
\|\hat{g}\|_{p}=\left\|\hat{w}_{k} * \hat{v}_{k} * \hat{g}\right\|_{p} \leqq\left\|\hat{w}_{k}\right\|_{1}\left\|\hat{v}_{k} * \hat{g}\right\|_{p} \leqq\left\|\hat{w}_{k}\right\|_{1}\|\hat{v} * \hat{g}\|_{p}=C\|\hat{v} * \hat{g}\|_{p} .
$$

Lemma 5. Let $p \neq 2$ and let $B$ be an open ball in $R^{n}$. Assume that 
$\lambda \in M_{p, B} \cap C^{2}(B)$, that $|\lambda|=1$ on $B$, and that there is a constant $C$ such that

$$
M_{p, B}\left(\lambda^{m}\right) \leqq C, \quad m=1,2, \ldots .
$$

Then there is an $x_{0} \in R^{n}$ and a complex number $c$ with $|c|=1$, such that

$$
\lambda(y)=c \exp \left(i\left\langle x_{0}, y\right\rangle\right), \quad y \in B .
$$

Proof. If $B=R^{n}$ this is Theorem 1.14 in [1]. We want to prove it for bounded $B$. Thereby we assume that it is already known that if $A$ is a real quadratic form and $\exp (i A) \in M_{p}, p \neq 2$, then $A=0$ (Lemma 1.4 in [1]).

Let $\lambda=\exp$ (if), $f$ be real and $f \in C^{2}(B)$. It will be sufficient to prove that the second order derivatives of $f$ vanish in $B$. Thus let $y_{0}$ be an arbitrary point in $B$. According to Lemma 3(ii) it is no restriction of the generality to assume that $y_{0}=0$. Let

$$
f(y)=f(0)+\left\langle x_{0}, y\right\rangle+A(y)+o\left(|y|^{2}\right), \quad y \rightarrow 0,
$$

where $A$ is a real quadratic form in $y$. Let

$$
g(y)=f(y)-f(0)-\left\langle x_{0}, y\right\rangle .
$$

Then, by Lemma 1(vi) and Lemma 3(iii)

$$
M_{p, B}(\exp (i m g)) \leqq C, \quad m=1,2, \ldots .
$$

Set $g_{m}(y)=m g\left(m^{-\frac{1}{b}} y\right)$. Then $g_{m} \rightarrow A$ uniformly on compact sets and also by Lemma 3 (ii),

$$
M_{p, m \frac{1}{b} B}\left(\exp \left(i g_{m}\right)\right)=M_{p, B}(\exp (i m g)) \leqq C
$$

By the above we can find a $\mu_{m} \in M_{p}$ such that $\mu_{m}=\exp \left(i g_{m}\right)$ on $m^{\frac{1}{3}} B$.

Let $B^{\prime}$ be a bounded open ball. Using Lemma 2 we see that there is a bounded open ball $B^{\prime \prime}$ and a function $k \in S$ such that $k=1$ on $B^{\prime}, k$ is zero outside $B^{\prime \prime}$ and $\|\hat{k}\|_{1} \leqq 2$. Choose $m_{0}$ so large that $m^{\frac{1}{2}} B \supset B^{\prime \prime}$, for $m \geqq m_{0}$. From Lemma 3(iii) we then get, $m>m_{0}$,

$$
M_{p}\left(\mu_{m} k\right) \leqq M_{p, m^{\frac{1}{2} B}}\left(\mu_{m}\right)\|\hat{k}\|_{1} \leqq 2 M_{p, m^{\frac{1}{2} B}}\left(\exp \left(i g_{m}\right)\right)
$$

and so, from the above

$$
M_{p}\left(\mu_{m} k\right) \leqq 2 C .
$$

Since $\mu_{m} k \rightarrow \exp (i A) k$ uniformly, Lemma $1(v)$ gives us

Hence

$$
M_{p}(\exp (i A) k) \leqq 2 C \text {. }
$$

$$
M_{p, B^{\prime}}(\exp (i A))=M_{p, B^{\prime}}(\exp (i A) k) \leqq M_{p}(\exp (i A) k) \leqq 2 C .
$$


Since the ball $B^{\prime}$ was arbitrary, Lemma 3 (iv) shows that $\exp (i A) \in M_{p}$ and so $A=0$, and the lemma is proved.

Lemma 6. Suppose that $A_{j}$ are Hermitian $N \times N$-matrices, $j=1, \ldots, n$, such that the eigenvalues (repeated with proper multiplicities) of

$$
\sum_{j=1}^{n} A_{j} y_{j}
$$

for all $y=\left(y_{1}, \ldots, y_{n}\right)$ in an open non-void ball $B$ in $R^{n}$ are of the form

$$
\sum_{j=1}^{n} \alpha_{k j} y_{j}, \quad k=1, \ldots, N,
$$

where the $\alpha_{k j}$ are constants. Then the matrices $A_{1}, \ldots, A_{n}$ commute.

Proof. When the conditions in Lemma 6 are satisfied for all complex $y_{j}$ (instead of $\left(y_{1}, \ldots, y_{n}\right) \in B$ ) this is a theorem by Motzkin and Taussky (Theorem 2 in [3]). From the analyticity of the both members in the equality

$$
\operatorname{det}\left(x E-\sum_{j=1}^{n} A_{j} y_{j}\right)=\prod_{k=1}^{N}\left(x-\sum_{j=1}^{n} \alpha_{k j} y_{j}\right), \quad y \in B,
$$

we see that it is also satisfied for all complex $y_{j}$, and Lemma 6 follows from the theorem of Motzkin and Taussky.

Proof of Theorem 1. Suppose $\mu(y)=\exp \left(i \sum_{j=1}^{n} A_{j} y_{j}\right)$ belongs to $\mathscr{M}_{p}$, $p \neq 2$. Since the elements of $\mu$ belong to $C^{\infty}\left(R^{n}\right)$ there is a non-void open ball $B$ in $R^{n}$ and functions $\lambda_{1}, \ldots, \lambda_{N}$ in $C^{\infty}\left(R^{n}\right)$ such that $\lambda_{1}(y), \ldots, \lambda_{N}(y)$ are the eigenvalues of $\mu(y)$, counted with proper multiplicities, for each $y \in B$, and such that for each $\lambda_{j}(y), y \in B$, there is an eigenvector $v_{j}(y) \neq 0$, and $v_{j} \in \mathscr{C}^{\infty}(B)$.

Since the behavior outside $B$ will be of no interest in the following, we can suppose that $v_{j} \in \mathscr{S}$ and that $\lambda_{j}$ on $B$ coincides with a function $f_{j} \in S$ (if necessary by shrinking the ball $B$ somewhat). Let $C_{j}$ be the constant associated with $v_{j}$ as in Lemma 4 , and we can suppose that the corresponding balls $B_{j}{ }^{\prime}$ are equal, to $B^{\prime}$ say. Let $g$ be any function in $S$ with $g=0$ outside $B^{\prime}$. Lemma 4 then gives

$$
\left\|\widehat{f}_{j}^{m} * \hat{g}\right\|_{p} \leqq C\left\|f_{j}^{m} * \hat{v}_{j} * \hat{g}\right\|_{p} .
$$

Since $g=0$ outside $B^{\prime}$ we have

$$
f_{j}^{m} v_{j} g=\lambda_{j}{ }^{m} v_{j} g=\mu^{m} v_{j} g .
$$

The inversion theorem and the inequality (3) then shows that

Math. Scand. $19-3$ 


$$
\left\|\widehat{f}_{j}^{m} * \hat{v}_{j} * \hat{g}\right\|_{p}=\left\|\widehat{\mu^{m}} * \hat{v}_{j} * \hat{g}\right\|_{p} \leqq \mathscr{M}_{p}\left(\mu^{m}\right)\left\|\hat{v}_{j}\right\|_{1}\|\hat{g}\|_{p} .
$$

Combination of (5) and (6) shows that

$$
\left\|\widehat{f_{j}^{m}} * \hat{g}\right\|_{p} \leqq C_{j} \mathscr{M}_{p}\left(\mu^{m}\right)\left\|\hat{v}_{j}\right\|_{1}\|\hat{g}\|_{p}
$$

Since $\mu^{m}(y)=\mu(m y)=m^{*} \mu(y)$, Lemma $1(\mathrm{iv})$ gives that

$$
\left\|{\widehat{f_{j}}}^{m} * \hat{g}\right\|_{p} \leqq C_{j} \mathscr{M}_{p}(\mu)\left\|\hat{v}_{j}\right\|_{1}\|\hat{g}\|_{p}=C_{j}^{\prime}\|\hat{g}\|_{p} .
$$

As $\mu$ is unitary we have $\left|\lambda_{j}\right|=1$, that is, $\left|f_{j}\right|=1$ on $B^{\prime}$. Since $f_{j} \in M_{p}$ (Lemma 1(vii)) we also have

$$
M_{p, B^{\prime}}\left(\lambda_{j}{ }^{m}\right)=M_{p, B^{\prime}}\left(f_{j}^{m}\right) \leqq C_{j}^{\prime}, \quad m=1,2, \ldots
$$

It follows that the conditions in Lemma 5 are satisfied, and from Lemma 6 we conclude that $A_{1}, \ldots, A_{n}$ commute.

To prove the converse we note that the Frobenius theorem shows that in this case $A_{1}, \ldots, A_{n}$ have a common diagonalization. It follows that there is a constant invertible matrix $P$ such that

where

$$
\exp \left(i \sum_{j=1}^{n} A_{j} y_{j}\right)=P\left(\exp \left(i \alpha_{k}(y)\right) \delta_{k l}\right) P^{-1}
$$

$$
\alpha_{k}(y)=\sum_{j=1}^{n} \alpha_{k j} y_{j}, \quad k=1, \ldots, N ; \alpha_{k j} \text { real constants } .
$$

By Lemma 1(vi) we have $\exp \left(i \alpha_{k}\right) \in M_{p}$ and so $\exp \left(i \sum_{j=1}^{n} A_{j} y_{j}\right)$ belongs to $\mathscr{M}_{p}$ and the theorem is proved.

\section{The initial value problem.}

We now turn to the Cauchy problem. Let $A_{j}(j=1, \ldots, n)$ and $B$ be $N \times N$-matrices and let $u=u(t, x)$ and $u_{0}=u_{0}(x)$ be $N$-dimensional complex vector functions. We consider the Cauchy problem

$$
\begin{cases}\frac{\partial u}{\partial t}=\sum_{j=1}^{n} A_{j} \frac{\partial u}{\partial x_{j}}+B u, & x \in R^{n}, \\ u(0, x)=u_{0}(x), & 0 \leqq t \leqq T .\end{cases}
$$

We say that the problem (1) is well posed in $L_{p}$ if for each $u_{0} \in \mathscr{S}$ there is a solution $u=u(t, x)$ of $(1)$ in $\mathscr{L}_{p}$-norm (by which we mean that

$$
\frac{1}{h}(u(t+h, x)-u(t, x)) \rightarrow \sum_{j=1}^{n} A_{j} \frac{\partial u}{\partial x_{j}}+B u
$$

in $\mathscr{L}_{p}$ when $h \rightarrow 0$ ) depending continuously (in $\mathscr{L}_{p}$ ) on the initial value $u_{0}$, 
i.e. there is a constant $C(T)$ such that

$$
\|u(t, \cdot)\|_{p} \leqq C(T)\left\|u_{0}\right\|_{p}, \quad 0 \leqq t \leqq T .
$$

Obviously such a solution is unique.

For $p=\infty$ this definition of well posed problems is weaker than the usual, since $\mathscr{S}$ is not dense in $\mathscr{L}_{\infty}$.

Our main result is Theorem 2.

Theorem 2. Suppose $p \neq 2,1 \leqq p \leqq \infty$. Then the Cauchy problem (1), where $A_{j}$ are Hermitian $N \times N$-matrices and $B$ is any $N \times N$-matrix, is well posed in $L_{p}$ if and only if the matrices $A_{1}, \ldots, A_{n}$ commute.

$B y$ the remarks above this gives a necessary condition also for the usual definition of well posed problems in $\mathscr{L}_{\infty}$.

Proof. Assume first that (1) is well posed in $L_{p}, p \neq 2$. Then since $u \in \mathscr{L}_{p}$, we can take the Fourier transforms, in the distribution sense, of the elements of (1) with respect to $x$ ( $t$ fixed) and get

$$
\left\{\begin{aligned}
\frac{\partial \hat{\imath}}{\partial t}(t, y) & =\left(-2 \pi i \sum_{j=1}^{n} A_{j} y_{j}+B\right) \hat{u}(t, y), & & y \in R^{n}, \\
\hat{u}(0, y) & =\hat{u}_{0}(y), & & 0 \leqq t \leqq T,
\end{aligned}\right.
$$

and so, with $\varphi_{t}(y)=\exp \left(t\left(i \sum_{j=1}^{n} A_{j} y_{j}+B\right)\right)$

$$
\hat{u}(t, y)=\varphi_{l}(-2 \pi y) \hat{u}_{0}(y) \text {. }
$$

Hence by (7) and Lemma 1(iv)

$$
\mathscr{M}\left(\varphi_{t}\right) \leqq C(T), \quad 0 \leqq t \leqq T .
$$

On the other hand, suppose $\left(^{*}\right)$ is satisfied. If $\varphi_{t}(-2 \pi y)=\hat{\mu}_{t}(y)$, then

$$
u(t, x)=\mu_{t} * u_{0}(x)
$$

and so $u(t, \cdot) \in \mathscr{C}^{\infty}\left(R^{n}\right)$ (the elements of $\mu_{t}$ are in $S^{\prime}$ and differentiation is continuous in $S)$. From

$$
\frac{\partial \hat{\mu}_{t}(y)}{\partial t}=\left(-2 \pi i \sum_{j=1}^{n} A_{j} y_{j}+B\right) \hat{\mu}_{t}(y), \quad \bar{\mu}_{0}(y)=E,
$$

it follows that

$$
\hat{\mu}_{l+h}(y)=\hat{\mu}_{t}(y)+\left(-2 \pi i \sum_{j=1}^{n} A_{j} y_{j}+B\right) h+h^{2} R_{2}(h ; t ; y),
$$

where the elements in $R_{2}$ are second order polynomials in $y$ with coefficients which are uniformly bounded in $M_{p}$, by $\left({ }^{*}\right)$ and Lemma 1(iv). 
Consequently there is a constant $C$ such that $\left\|\frac{1}{h}(u(t+h, \cdot)-u(t, \cdot))-\left(\sum_{j=1}^{n} A_{j} \frac{\partial u}{\partial x_{j}}(t, \cdot)+B u(t, \cdot)\right)\right\|_{p} \leqq|h| C \sum_{|\alpha| \leqq 2}\left\|D^{\alpha} u_{0}\right\|_{p}$, and so $u$ is a solution of (1) in $\mathscr{L}_{p}$-norm. By Lemma $1(\mathrm{iv})$ and (*)

$$
\|u(t, \cdot)\|_{p}=\left\|\mu_{t} * u_{0}\right\|_{p} \leqq C(T)\left\|u_{0}\right\|_{p} .
$$

Hence (1) is well posed in $L_{p}$.

By Theorem 1, the following lemma then completes the proof of Theorem 2.

Lemma 7. Let $A_{j}(j=1, \ldots, n)$ and $B$ be $N \times N$-matrices and let

Then

$$
\varphi_{t}(y)=\exp \left(t\left(i \sum_{j=1}^{n} A_{j} y_{j}+B\right)\right)
$$

$$
\mathscr{M}_{p}\left(\varphi_{t}\right) \leqq C(T), \quad 0 \leqq t \leqq T,
$$

if and only if $\exp \left(i \sum_{j=1}^{n} A_{j} y_{j}\right)$ belongs to $\mathscr{M}_{p}$.

Proof. Suppose first that (8) holds. Then, by Lemma 1(iv), $\psi_{t}(y)=$ $\exp \left(i \sum_{j=1}^{n} A_{j} y_{j}+t B\right)$ satisfies

$$
\mathscr{M}_{p}\left(\psi_{t}\right) \leqq C(T), \quad 0<t \leqq T .
$$

If we let $t \rightarrow 0$, we see that $\psi_{t}(y) \rightarrow \exp \left(i \sum_{j=1}^{n} A_{j} y_{j}\right)$ uniformly on compact subsets of $R^{n}$, and so Lemma $1(\mathrm{v})$ shows that $\exp \left(i \sum_{j=1}^{n} A_{j} y_{j}\right)$ belongs to $\mathscr{M}_{p}$.

On the other hand, if $\mu(y)=\exp \left(i \sum_{j=1}^{n} A_{j} y_{j}\right)$ is in $\mathscr{M}_{p}$, then by Lemma l(iv)

$$
\mathscr{M}_{p}\left((t)^{*} \mu\right) \leqq \mathscr{M}_{p}(\mu), \quad 0 \leqq t \leqq T .
$$

Let $D$ be a bounded open ball in $R^{n}$. Then $\varphi_{t} \in \mathscr{M}_{p, D}, 0 \leqq t \leqq T$. The elements of $\varphi_{t}$ and their derivatives are bounded on compact subsets of $R^{n}$, uniformly for $0 \leqq t \leqq T$. If we multiply $\varphi_{t}$ by a function $k_{D} \in C^{\infty}\left(R^{n}\right)$ with value 1 on $D$ and with compact support, then $k_{D} \varphi_{t}$ has elements belonging to $S$ which, together with their derivatives, are uniformly bounded in $L_{1}$ for $0 \leqq t \leqq T$. Hence $\mathscr{M}_{p, D}\left(\varphi_{t}\right) \leqq \mathscr{M}_{p}\left(k_{D} \varphi_{t}\right)$ is uniformly bounded for $0 \leqq t \leqq T$.

We also note that $\varphi_{t}$ is a solution of

$$
\frac{\partial \varphi_{l}}{\partial t}(y)=\left(i \sum A_{j} y_{j}+B\right) \varphi_{t}(y), \quad \varphi_{0}(y)=E,
$$


that is,

$$
\varphi_{t}(y)=\mu(t y)+\int_{0}^{t} \mu((t-v) y) B \varphi_{v}(y) d v
$$

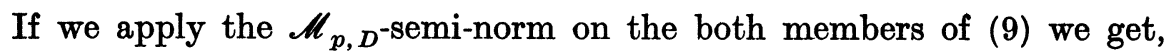
using Lemma 3(iii), Lemma 1 (iv) and the remarks above, that

$$
\mathscr{M}_{p, D}\left(\varphi_{t}\right) \leqq \mathscr{M}_{p}(\mu)+\mathscr{M}_{p}(\mu) \int_{0}^{t}|B| \mathscr{M}_{p, D}\left(\varphi_{v}\right) d v,
$$

and so, since the integral is bounded by the remarks about $\varphi_{t}$ above, Gronwall's lemma applies:

$\mathscr{M}_{p, D}\left(\varphi_{t}\right) \leqq \mathscr{M}_{p}(\mu) \exp \left(\mathscr{M}_{p}(\mu) \int_{0}^{t}|B| d v\right) \leqq \mathscr{M}_{p}(\mu) \exp \left(T|B| \mathscr{M}_{p}(\mu)\right), 0 \leqq t \leqq T$. By Lemma 3(iv) then

$$
\mathscr{M}_{p}\left(\varphi_{t}\right) \leqq \mathscr{M}_{p}(\mu) \exp \left(T|B| \mathscr{M}_{p}(\mu)\right)=C(T), \quad 0 \leqq t \leqq T,
$$

and Lemma 7 is proved.

\section{REFERENCES}

1. L. Hörmander, Estimates for translation invariant operators in $L_{p}$ spaces, Acta Math. 104 (1960), 93-140.

2. W. Littman, The wave operator and $L_{p}$ norms, J. Math. Mech. 12 (1963), 55-68.

3. T. S. Motzkin and O. Taussky, Pairs of matrices with property L, Trans. Amer. Math. Soc. 73 (1952), 108-114.

DEPARTMENT OF MATHEMATICS, CHALMERS INSTITUTE OF TECHNOLOGY AND THE UNIVERSITY OF GOTEBORG, GÖTEBORG, SWEDEN 